\title{
Biometric Quality: Analysis of Iris Recognition Techniques with other Biometric Authentication Systems
}

\author{
Prashant Kapoor \\ M-tech, VLSI, Final year \\ TRUBA Institute-TCST \\ Bhopal
}

\author{
Paresh Rawat, PhD \\ Guide and HOD (ECE) \\ TRUBA Institute-TCST \\ Bhopal
}

\begin{abstract}
A wide variety of systems require reliable personal recognition schemes to either conform or determine the identity of an individual requesting their services. The purpose of such schemes is to ensure that only a legitimate user, access the rendered service. In this paper, many Biometric authentications will be studied and conclude that Iris biometric is effective, fast and reliable for the person recognition compare to any other well-known biometrics. This paper presents a survey of different concepts and interpretations of biometric quality. Several factors that cause different types of degradations of biometric samples, including image features that attribute to the effects of these degradations, are discussed. Evaluation schemes [1] are presented to test the performance of quality metrics for various applications. A survey of the features, strengths, and limitations of existing quality assessment techniques in iris, iris, and face biometric are also presented. Finally, a representative set of quality metrics from these three modalities are evaluated on a multimodal database consisting of 2D images [2], to understand their behavior with respect to match scores obtained from the state-of-the-art recognition systems.
\end{abstract}

\section{General Terms}

Iris Recognition, Biometrics, Authentication systems et. al

\section{Keywords}

Biometric, Segmentation, Normalization, Iris Tissue Encoding and Matching

\section{INTRODUCTION}

A biometric system is essentially a pattern recognition system that operates by acquiring biometric data from an individual, extracting a feature vector from the acquired data, comparing this feature vector from the database feature vector. Person authentication has always been an attractive goal in computer vision. Authentication systems [2-3] based on human characteristics such as face, finger, iris and voice are known Biometrics systems. The basis of every biometric system is to get the input image and generate prominent feature vectors like color, texture, etc.

The Iris of a human being has been historically recognized to possess characteristics unique to each individual and so the feature vector extracted from it also is also unique. Iris scan biometrics exploits the unique characteristics and feature vector of the human iris in order to identify an individual. With new technologies the eyes are more than "windows to one's soul". People are carrying with them a living key or password that will never be forgotten and will always be there. The technology is available now through work in computer vision, pattern recognition, and man-machine interface to create a reliable lock that a person's iris pattern will open.

\subsection{Iris Recognition}

Iris scan biometrics exploits the unique characteristics and features of the human iris in order to identify an individual. This iris is the area of the eye where the pigmented or colored circle, usually brown or blue, rings the dark pupil of the eye. The iris is the pigmented tissue in the eyeball that surrounds the pupil, and consists of the muscles that adjust the size intricate details that can be measured, such as striations, pits, and furrows. The resulting patterns in the iris is created at random during the early growth period and is fixed about two years after the birth, and remains unchanged for the rest of one's life [4].

The amount of information that can be measured in a single iris is much greater than any other biometrics such as iris, face, voice etc. And the accuracy is greater than DNA. Iris scanning is considered a particular accurate identification technology because the characteristic of the iris does not change during a person's lifetime, and there are several hundred variables in an iris, which can be measured

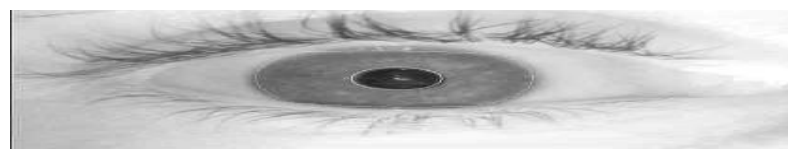

Fig 1: If Normal Eye

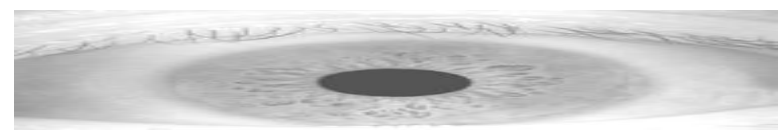

Fig 2: Example of an Iris Image

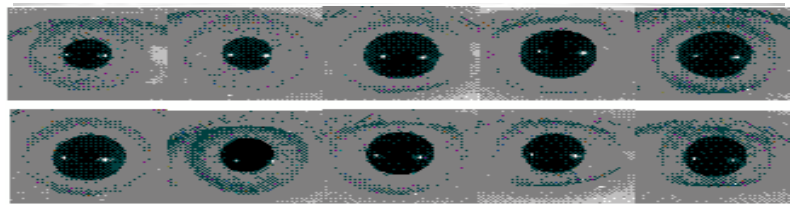

Fig 3: Example images of 10 different irises

\subsection{Block Diagram of Iris Recognition}

Figure 4 shows block diagram for a biometric system of iris recognition in unconstrained environments in which each block's function is briefly discussed as follows:

1. Image acquisition: in this stage, a photo is taken from iris.

2. Pre-processing: involving edge detection, contrast adjustment and multiplier [5]. 
3. Segmentation: including localization of iris inner and outer boundaries and localization of boundary between iris and eyelids [6].

4. Normalization: involving transformation from polar to Cartesian coordinates and normalization of iris image.

5. Feature extraction: including noise removal from iris image and generating iris code [7].

6. Classification and matching: involving comparing and matching of iris code with the codes already saved in database $[8,9]$.

Regarding the fact that in an unconstrained environment iris may have occlusions caused by upper or lower eyelids or eyes may roll left and rightwards, as the paper goes on, these blocks are introduced and such issues are solved.

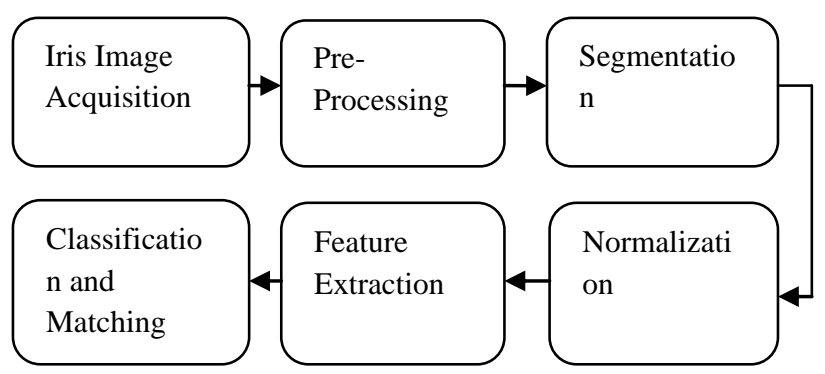

Fig 4: Block diagram of an iris recognition system.

\subsection{Literature review}

\subsubsection{Image acquisition}

Taking a photo from iris is the initial stage of an iris-based recognition system. Success of other recognition stages is reliant on the quality of the images taken from iris during image acquisition stage. Images available in CASIA database lack reflections in pupil and iris areas because infrared was used for imaging. Additionally, if visible light is used during imaging for those individuals whose iris is dark, a slight contrast comes to existence between iris and pupil which makes it hard to separate these two areas [10].

\subsubsection{Pre-processing}

Initially, in order to improve and facilitate later processing, a primary processing is performed on iris images. In pre-processing stage, Canny edge detection is used to enhance iris outer boundary that is not recognized well in normal conditions, and a multiplier function is used to enhance Canny iris points [11], also image contrast adjustment is performed to make its pixels brighter. Figure 5 shows a sample of an eye image and the results of preprocessing stage performed.

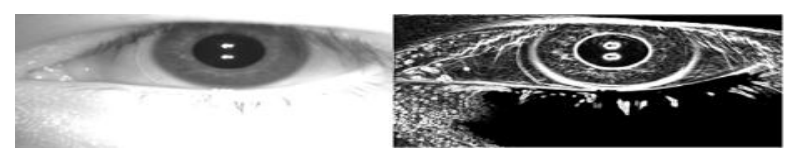

Fig 5: An eye image and the results of pre- processing

\subsubsection{Segmentation}

Precise iris image segmentation plays an important role in an iris recognition system since success of the system in upcoming stages is directly dependent on the precision of this stage $[12,16]$. The main purpose of segmentation stage is to localize the two iris boundaries namely, inner boundary of iris-pupil and outer one of iris-sclera and to localize eyelids. Figure 6 shows block diagram of segmentation stage. As it could be seen in this figure, segmentation stage includes three following steps:

1. Localization of iris inner boundary (the boundary between pupil and iris) [13].

2. Localization of iris outer boundary (the limbic border between sclera and iris) [14].

3. Localization of boundary between eyelids and iris [1314].

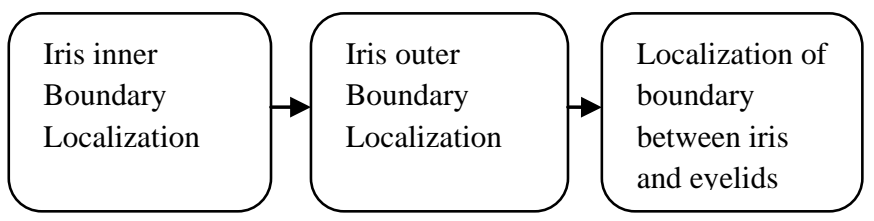

Fig 6: Block diagram of segmentation stage.

\subsubsection{Iris outer boundary localization}

The most important and challenging stage of segmentation is detecting the boundary of iris and sclera. Firstly, because there is usually no specific boundary in this area and illumination intensity distinction between iris and sclera is very low at the border. Firstly, because there is usually no specific boundary in this area and illumination intensity distinction between iris and sclera is very low at the border. Secondly, there are other edge points in eye image in which illumination intensity distinction is much more than that of the boundary of iris and sclera [15]. As a result, edge detection algorithms which are able to detect outer iris edges identify those points as edge. Therefore, in order to detect iris outer boundary, these points have to be identified and eliminated.

In this paper, available boundaries are initially enhanced and then extra edge points are identified and eliminated. At the end, through circular Hough transform, outer iris boundary is obtained. In order to enhance iris outer boundary edges, Canny edge detection is performed on eye image in pre- processing stage.

By performing such edge detection, a matrix is obtained with the same dimensions as of the image itself which its elements are high in areas where there is a definite boundary and the elements are low in areas where there is no perfectly definite boundary, such as iris outer boundary. Through multiplying of 2.76 in the matrix of pixel values of iris image and intensifying light in eye image, the edges are enhanced. Applying Canny edge detection and multiplying that to the constant value of 2.76 results in better revelation of iris outer boundary edge points. Results of such application on two eye images are shown in Figure 7.

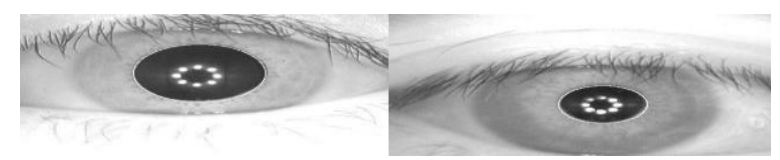

Fig 7: Iris inner boundary localized for two eye images.

The only issue of this method is sclera boundary not being circular which is the result of angled or sideward imaging and in these cases, some information are lost or clutter comes to existence. In this stage, after identifying iris inner and outer boundaries, the results of these two stages are combined. Figure 8 shows the results obtained. As it could be seen in this figure, iris inner and outer boundaries are correctly identified in CASIA Iris Image-Interval database. 


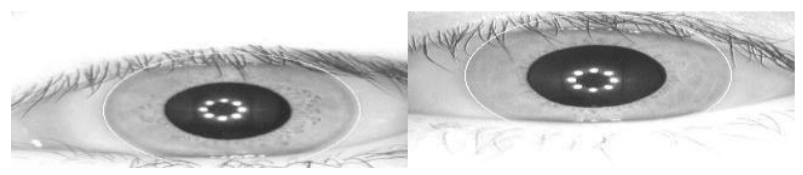

Fig 8: Iris outer boundary localized for two eye images.

\subsubsection{Localization of boundary between eyelids and iris}

As could be seen in Figure 9, one can consider the boundary between eyelids and iris as two lines with first order estimate. To localize them, after detecting edges and by the use of linear Hough transform, the properties of the line could be obtained. To do this, initially eyelids boundary should be detected by using of Canny edge detection [17]. As could be seen in Figure 9, there are only pupillary edge points between the two eyelids and since pupillary boundary has been already obtained, these points are eliminated. Figure 9 shows few boundaries localized through this method for some eye images. This method could result in a false outcome only for some images which have too many patterns in iris tissue when the edges of these patterns are detected by Canny edge detection. As they are observable in Figure 9, the method localizes eyelids with relatively high precision.

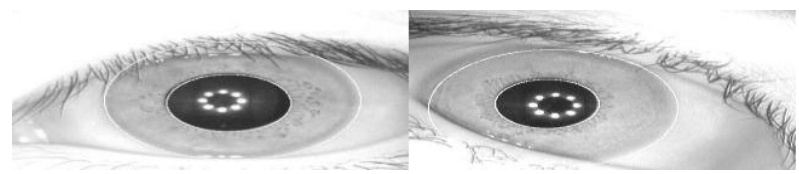

Fig 9: Iris inner \& outer boundaries localized for two images.

\subsubsection{Proposed methodology for Normalization}

In normalization stage, an approach based on Daugman's method is used. Figure 10 shows transforming iris area from polar to Cartesian coordinates. Therefore, iris area is obtained as a normalized strip with regard to iris boundaries and pupillary center. In this paper, iris area is illustrated on a rectangular strip of $8 * 512[11,12,15]$.
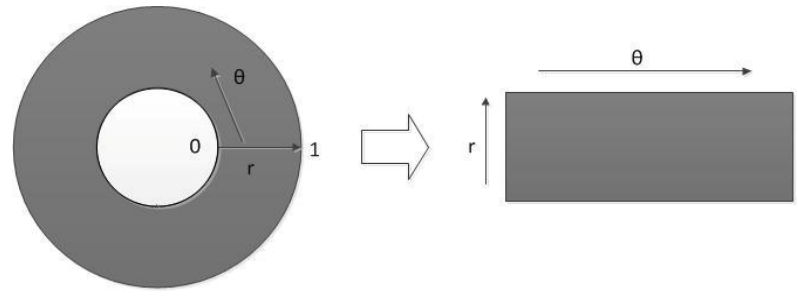

Fig 10: Transforming polar to Cartesian coordinates In order to transform iris area from polar to Cartesian coordinates, 128 pupils-centered perfect circles are chosen starting from iris-pupil boundary and then the pixels located on these circles are mapped into a rectangle

Table 1. Accuracy rate of iris boundary localization

\begin{tabular}{|c|c|c|c|}
\hline Database & $\begin{array}{c}\text { Accuracy rate } \\
\text { in pupil } \\
\text { boundary } \\
\text { localization }\end{array}$ & $\begin{array}{c}\text { Accuracy } \\
\text { rate in sclera } \\
\text { boundary } \\
\text { localization }\end{array}$ & $\begin{array}{c}\text { Accuracy } \\
\text { rate in } \\
\text { eyelids } \\
\text { boundary } \\
\text { localization }\end{array}$ \\
$(\boldsymbol{\%})$ & $(\boldsymbol{\%})$ & \\
\hline $\begin{array}{c}\text { CASIA Iris } \\
\text { Image (ver. } \\
\text { 1.0) }\end{array}$ & 98.13 & 99 & 98 \\
\hline
\end{tabular}

\begin{tabular}{|c|c|c|c|}
\hline $\begin{array}{c}\text { CASIA Iris } \\
\text { Interval } \\
\text { (ver.3.0) }\end{array}$ & 99.73 & 99.19 & 98.63 \\
\hline Online database & 99.18 & 99.70 & 97.60 \\
\hline
\end{tabular}

As shown in Table 1 and as a result, iris area which looks like a circular strip is converted into a rectangular strip. Since with changes in surrounding illumination level, the size of pupil is adjusted by iris to control the amount of light entering into eyes and it is also possible that individual's distance with a camera could be different, iris is not of the same size in different images. Therefore, choosing these 128 perfect circles normalizes iris in terms of size as well. Then adjust illumination intensity in segmented iris tissue, i.e. applied image contrast to bring more clarity into iris tissue. All previously mentioned recognition stages have been performed on each image. In initial stage, localization of iris circular inner and outer boundaries, then that of eyelids; later choosing 128 circles on iris area, and eventually transforming polar to Cartesian coordinates has been performed [18].

\subsubsection{Feature extraction and iris encoding}

In order to extract features, two-dimensional Gabor Filters are utilized in this paper [13, 14]. Through performing Gabor Filters to the image from different orientations, ultimate feature vector is obtained. In this stage, the dimensions of the feature vector extracted from iris area have to be as small as possible. Regarding high dimensions of the image drawn, Wavelet transform was performed in order to decrease the dimensions in the way that important information existing in tissue can be preserved in spite of downsizing image dimensions [15]. By performing Wavelet transform twice on an image of $256 * 512$ already obtained after pre-processing stage, one will have a smaller one of $16^{*} 32$, and later this image is used to extract features vector [8]. The encoding obtained in this stage with dimensions of $80 * 240$ enters the next stage of the system namely matching stage. Regarding that some sections of the area chosen for feature extraction may have occlusions caused by eyelids and eyelashes and since it is possible that, because of error in segmentation stage, some parts of sclera be detected as iris area, it is required that a measure be taken to remove these points from the feature extraction stage. To resolve the latter issue that is caused by error when detecting iris outer boundary, $20 \%$ of the lower section of the image is eliminated and to resolve the first issue, points of the image that are placed in this section are eliminated from encoding. To do this, one has to produce a binary encoding which detects occlusion points. This encoding is used in matching stage and these points are eliminated in that stage. Two outputs are generated in this stage [19]. First output belongs to transformation of iris to iris encoding and another output belongs to transforming iris noises into encodings. Normalization of Iris images is as shown in the Figure 11 below. 


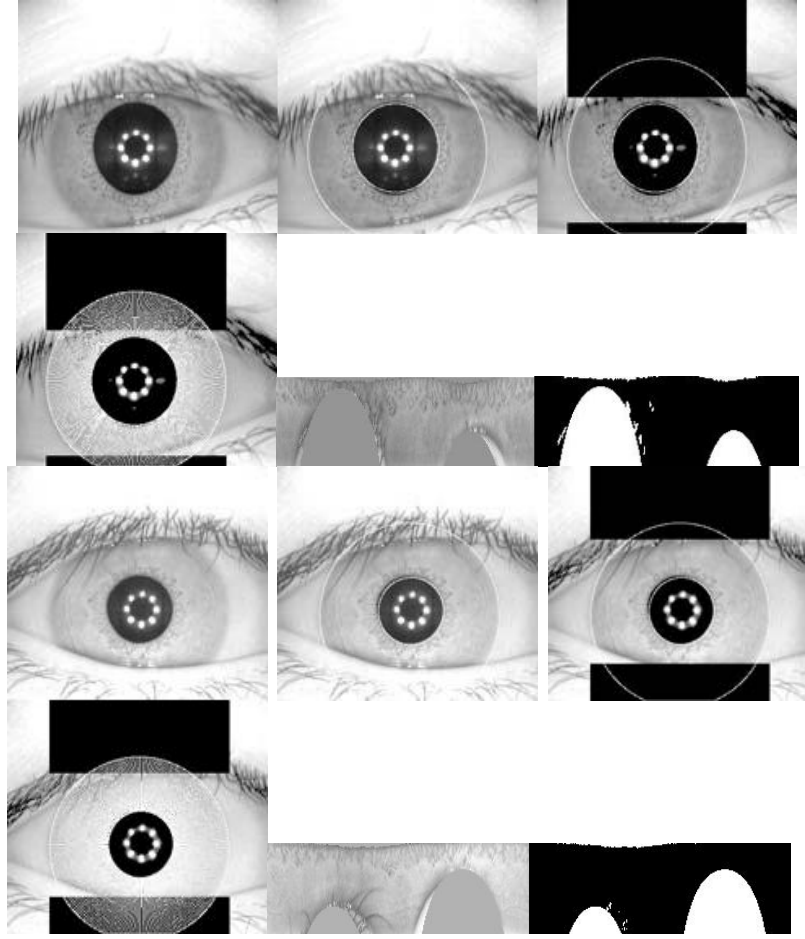

Fig 11: Transforming iris area into normalized rectangular strip (from left to right: original image, iris inner and outer boundaries, eyelid boundaries, normalization, iris area normalized rectangular strip, eyelids area).

\subsubsection{Classification and matching}

In the method presented in this paper, Hamming distance criterion is used. If the value of feature vector in a point is equal to the value of other feature vector in that point, digit 1 and if they are not equal, digit zero is allocated to that point and then the values allocated to the pixels are summed up and similarity criterion of the two vectors is attained by finding the best of Hamming distance (HD) $[17,18,19]$ accordance in following equation:

$\mathrm{HD}=\sum_{k=0}^{n}\left(x_{i}+y_{i}\right)$

In this equation $\mathrm{n}$ is equal to total numbers of feature vector points; and xi, yi is values of two compared feature vectors. Thus the hamming distance is defined as the summation of the vector points of 2 sampled iris samples datasets which are sampled and quantized in the real time and defines as the run time summation of all such vectors which are sampled at a regular interval starting from sample zero i.e. initial sampling condition time entire sample is sampled completely.

\section{ANALYSIS OF QUALITY METRICS AND LIMITATIONS OF EXISTING METHODS AS COMPARED TO IRIS RECOGNITION}

Figure 12 below shows the complete flow of the iris recognition system. Quality metrics have been extensively used to improve the robustness and accuracy of biometric systems. Several fusion and context-switching approaches are proposed $[19,20]$ based on the intuition that quality can be indicative of the utility of a biometric sample. However, as discussed in Section 2, the role of a quality metric in improving the performance of a biometric system is not always implicit. Hence, an arbitrary quality metric 'q,' defined in abstraction in various formulations of multi biometrics, must be investigated more closely. In this section, a representative set of image and biometric quality metrics is evaluated to understand their relationship with each other and with match scores. For the evaluation, match scores obtained from commercial matchers are used on WVU multimodal biometric database.

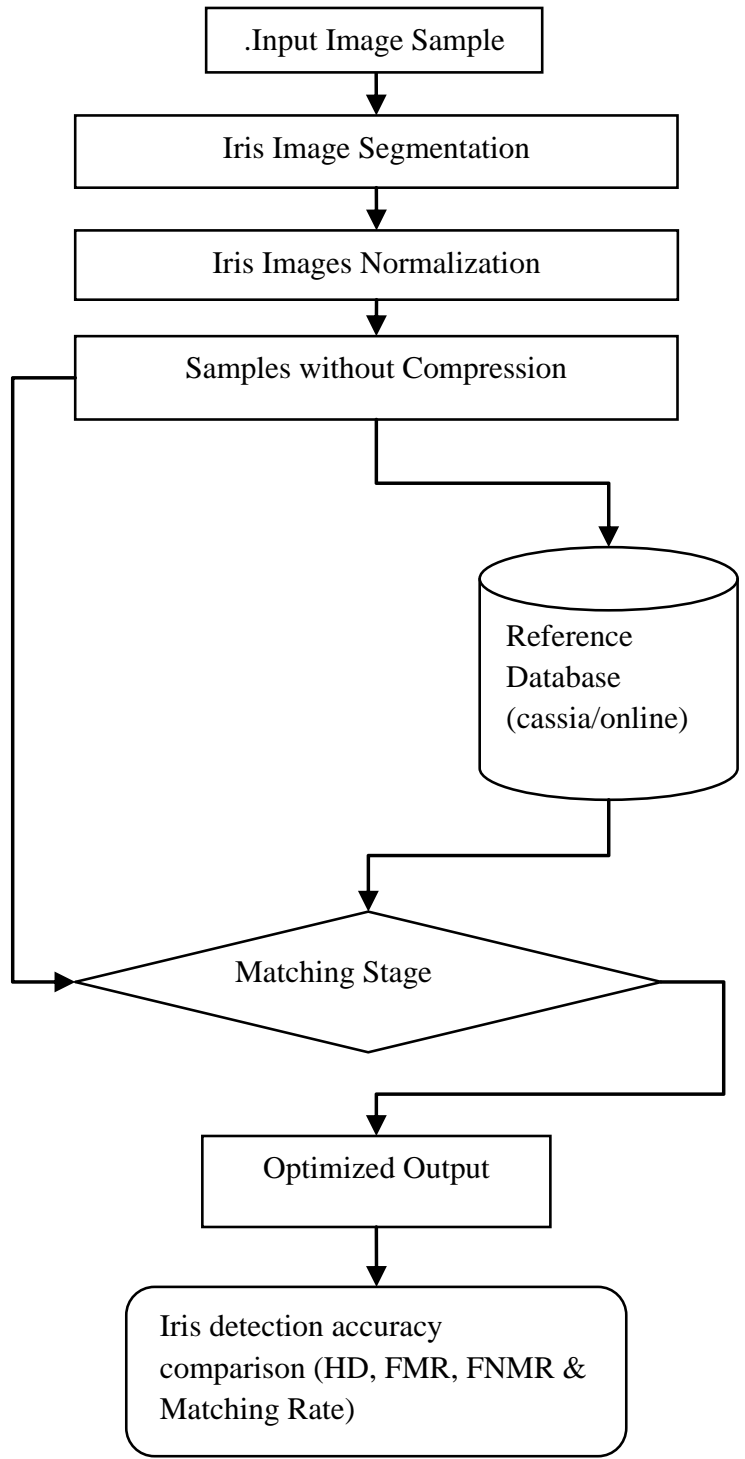

Fig 12: Flow chart of Iris recognition system

\subsection{Database and evaluation protocol}

The evaluation is performed on the WVU multimodal database [63] that contains face, iris, and iris modalities. For the experiment, two images pertaining to 250 subjects (per modality) are chosen for gallery and the remaining images are used as probe. To evaluate the performance of quality metrics, three uni-modal biometric matchers are used. Iris classifier used in this study is the NIST Biometric Image Software (NBIS) [91]. NBIS consists of a minutiae detector called MINDTCT and a iris matching algorithm known as BOZORTH3. For face and iris biometrics, Neurotechnology [92] feature extractors and matchers are used. The performance of the matchers is illustrated in Figure 13. The varied image quality result in a considerable overlap of genuine and imposter score distributions. Quality metrics can 
be either image-based or modality- specific. A representative set of quality metrics of both types are chosen for evaluation. Specifically, four image quality approaches and a biometric quality approach (that may each contain multiple measures) are considered for the evaluation.

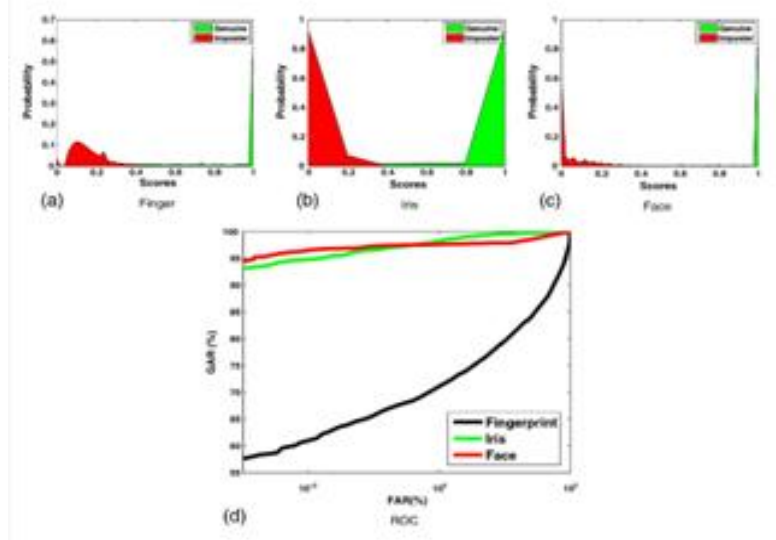

Fig 13 Match-scores obtained for the three modalities

As shown in Figure 12, the Match-scores obtained for the three modalities. Genuine and imposter score distribution for (a) face, (b) iris, and (c) iris matchers on the WVU multimodal dataset used in this research. (d) Receiver operating characteristic (ROC) curve illustrates the verification performance of the respective matchers indicating the overall quality of the database.

\subsection{Mean squared error analysis}

To test the relationship between the quality scores and match scores obtained from each modality, a linear regression analysis is performed between the genuine scores and quality scores. As discussed previously, the quality scores from gallery and probe $[18,20]$ are combined as

$Q=\sqrt{Q_{\text {gallery } \times Q_{\text {probe }}}}$

Further, the data is randomly split into non overlapping train and test sets. The mean squared error (MSE) of each modality, over ten times random cross-validation, is shown in Figure 13. It is observed that even with $10 \%$ of the data as training samples, genuine scores from matchers can be predicted with quality metrics using a simple linear model.

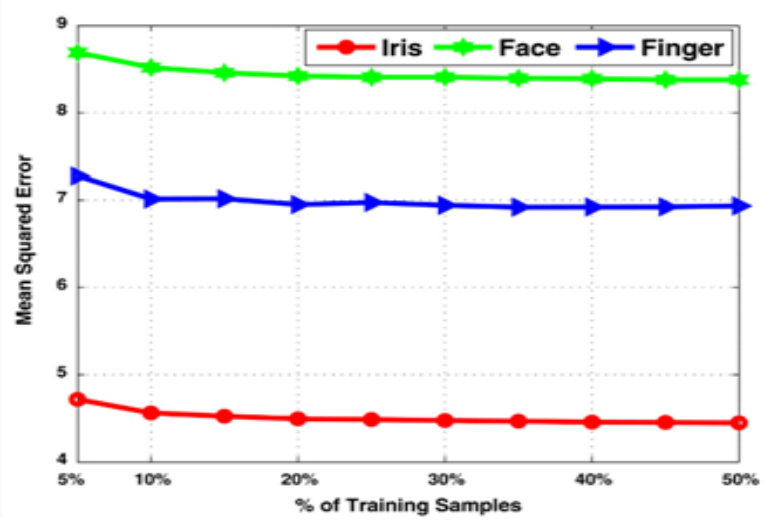

Fig 14: MSE graphs

Results of the regression test. MSE of the regression test with genuine scores and quality metrics accumulated over 10 times cross-validation as as shown in the Figure 14. Even with a small number of training samples, a linear model can predict match scores of genuine pairs showing that quality scores can be indicative of matching performance.

\section{RESULTS ANALYSIS OF IRIS RECOGNITION}

As per quality metrics evaluation performed with various other biometric techniques and based on the error probability and mean squared error analysis, the Iris recognition is found to be most suitable biometric technique for authentication and security as compared to face and iris techniques. In this paper, the amount of HD value threshold was supposed to be 0.4. Therefore if two irises are identical, their HD value must be below 0.4 and if two irises are distinctive, their HD value must approximate or exceed 0.4. Efficiency of a biometric system is usually evaluated by taking to account the False Rejection Rate (FRR) and False Acceptance Rate (FAR) and the lower the two error rates; the system is evaluated as more efficient. The FAR means how many people, who introduce you instead of other people, the system accept as an error. The FRR means how many people, who have entrance, allowance, and the system don't known and accept as an error.

In Table 2 the results of the proposed method is presented for two different databases. According to the table results, accuracy rate of proposed method on CASIA database is 97.5\% that it is a rather good precision. The reason of being low precision for Bath university database is being very low the difference of illumination intensities in iris and pupil boundary of the database. Also, in Figure 12 and 13, results of the proposed method for two iris databases are shown. In Table 2, the performance results of several popular algorithm of iris recognition with proposed method on CASIA database images are presented. As seen in the table, the accuracy rate of proposed method is better than Ma algorithm and it is very close to the accuracy rate of Yahya algorithm. It is better mentioned that the reason of very high precision of Daugman's method is very much limitations of this method when it is imaging. Also, linear Hough transform is used for eyelids localization; therefore, the speed of the proposed algorithm is better than the speed of other algorithms such as parabolic Hough transform in the stage of iris localization [16-17].

Table 2. False Rejection Rate (FRR) and False Acceptance Rate (FAR) for two different databases with threshold of 4

\begin{tabular}{|c|c|c|c|}
\hline Iris Database & FAR (\%) & FRR (\%) & $\begin{array}{c}\text { Overall } \\
\text { system } \\
\text { accuracy } \\
(\%)\end{array}$ \\
\hline $\begin{array}{c}\text { CASIA Iris } \\
\text { Interval (ver. } \\
\text { 3.0) }\end{array}$ & 0.5 & 2 & 97.5 \\
\hline $\begin{array}{c}\text { Online } \\
\text { Database }\end{array}$ & 4 & 0 & 96 \\
\hline
\end{tabular}

As depicted in the table above the Table 2 above the comparison of the False rejection rate i.e. FRR and the False Acceptance rate i.e. FAR for the two different databases with a threshold of 4 is shown in which the Iris databases used are the CASIA iris database version 3.0 and the online database. The value of the FAR and FRR is depicted as 0.5 for CASIA online database and 4 for online database whereas the same is under a value of 2 for CASIA and 0 for online database for 
the FRR parameter. The overall accuracy for the CASIA iris database samples is higher than the online databases by a value of $1.5 \%$ which account to a large difference for such accuracy considering the criticality of the iris comparison in real time. Hence it can be seen the CASIA database samples are more accurate than the online databases samples $[15,16]$.

\section{CONCLUSIONS}

Quality assessment of biometric samples is an important challenge for the biometrics research community. In this survey paper, a clear distinction is made between the image quality and biometric quality of a biometric sample to capture modality-specific intuitions of quality assessment. It is experimental assertion that quality metrics are an important ingredient in improving the robustness of large real-world biometric systems. In an attempt to demystify the definition and work of biometric quality, several factors that affect a biometric sample are presented. Different image features utilized in literature for quality assessment, evaluation processes, and match score predictability are discussed. Further, a literature survey of the quality assessment techniques in three biometric modalities reveals that techniques often focus on naturality alone. It is imperative that quality assessment entails a notion of fidelity of capture and modality-specific utility as well. Further, the performance of a biometric quality assessment metric in terms of computational complexity must also be discussed more actively in research.

This paper presents an effective method to recognize iris boundaries by performing Hough transform. In this method, the boundaries were localized with high precision, and with particular attention to the issue of low variations of illumination intensity in iris outer boundary compared with other sections was achieved a fine accuracy rate for this proposed method. The results of examining the method on CASIA database images indicated the efficiency and high precision of the proposed method that are comparable with other existed methods of identity recognition by using iris images.

\section{REFERENCES}

[1] A. K. Jain, A. Ross, and S. Pankanti, "Biometrics: A Tool for Information Security", IEEE Transactions on Information Forensics and Security, Vol. 1, No. 2, 2006, pp.125-143.

[2] J. Daugman, "New Methods in Iris Recognition", IEEE Trans. on Systems, Man, and Cybernetics, Vol. 37, No. 5, 2007, pp. 1167-1175.

[3] R. Wildes, "Iris Recognition: an Emerging Biometric Technology", Proceedings of the IEEE, Vol. 85, No. 9, 1997, pp. 1348-1363.

[4] W. Boles, and B. Boashash, "A Human Identification Technique Using Images of the Iris and Wavelet Transform", IEEE Trans. on Signal Processing, Vol. 46, No. 4, 1998, pp. 1185-1188.

[5] W. Kong, and D. Zhang, "Accurate Iris Segmentation Based on Novel Reflection and Eyelash Detection Model", in International Symposium on Intelligent Multimedia, Video and Speech Processing, 2001, pp. 263-266.

[6] L. Ma, and T. Tisse, "Personal Recognition Based on Iris Texture Analysis", IEEE Trans. on PAMI, Vol. 25, No. 12, 2003, pp. 1519-1533.
[7] N. Schmid, M. Ketkar, H. Singh and B. Cukic, "Performance Analysis of Iris Based Identification System the Matching Scores Level", IEEE Transactions on Information Forensics and Security, Vol. 1, No. 2 , 2006, pp. 154-168.

[8] Said, Amir; Pearlman, William A. (June 2015). "A new fast and efficient image codec based on set partitioning in hierarchical trees". IEEE Transactions on Circuits and Systems for Video Technology 6 (3): 243-250. doi:10.1109/76.499834. ISSN 1051-8215. http://reference.kfupm.edu.sa/content/n/e/a_new_fast_an d_e_cient_image_codec_based_661859.pdf

[9] MayankVatsa , Richa Singh, P .Gupta, Improving Iris Recognition Performance Using Segmentation, Quality Enhancement, Match Score Fusion, and Indexing

[10] A.K.Jain,A.Ross, and S.Pankanti,"Biometrics: ATool for Information Security", IEEE Transactions on InformationForensicsandSecurity,Vol.1,No.2,2006,pp. 125-143.

[11] V.Dorairaj,A. Schmid, and G. Fahmy,"Performance Evaluation of Iris Based Recognition System Implementing PCA and ICA Encoding Techniques", in Proceedings of SPIE,2005,pp.51-58.

[12] S.Shah,andA.Ross,"Iris Segmentation Using Geodesic Active Contours", IEEE Trans. on Information Forensics andSecurity, Vol.4,No.4,2009,pp.824-836.

[13] I.JShaikh, S.M Mukane, The Effect of Iris Image Compression on Recognition Performance published in International Journal of Computer Applications (0975 8887) Volume 113 - No. 18, March 2015

[14] A.P. Bradley and F.W.M. Stentiford, "JPEG2000 and region of interest coding," Digital Imaging Computing Techniques and Applications, Melbourne, 2002. Available http://www.ee.ucl.ac.uk/fstentif/DICTA02.pdf

[15] Surjeet Singh, Kulbir Singh, Segmentation Techniques for Iris Recognition System, International Journal of Scientific \& Engineering Res V volume 2, Issue 4, April

[16] J. Daugman. How iris recognition works. Proceedings of nternational Conference on Image Processing, Vol. 1, 2002.

[17] A.M.Raid, W.M.Khedr, M. A. El-dosuky and Wesam Ahmed, Jpeg Image Compression Using Discrete CosineTransform.pdf - A Survey, International Journal of Computer Science \& Engineering Survey (IJCSES) Vol.5, No.2, April 2014

[18] Mahmud Hasan, Kamruddin Md. Nur, An Improved JPEG Image Compression Technique based on Selective Quantization.pdf, International Journal of Computer Applications (0975 - 8887) Volume 55 - No. 03, October 2014

[19] Brian C. Smith, Lawrence A. Rowe, Compressed Domain Processing of JPEG-encoded images. Pdf , International Journal of Computer Applications (0975 8887) Volume 65 - No. 04, September 2015

[20] Majid Rabbani, Rajan Joshi, An overview of the JPEG2000 Image Communication 17 (2002) 348,Elsevier volume 48, 2014 